ARTICLE

DOI: $10.1038 / s 41467-018-07095-z$

\title{
Dehydrative $\pi$-extension to nanographenes with zig-zag edges
}

\author{
Dominik Lungerich (10 1,2, Olena Papaianina ${ }^{1}$, Mikhail Feofanov (10 ${ }^{1}$, Jia Liu ${ }^{3}$, Mirunalini Devarajulu ${ }^{3}$
} Sergey I. Troyanov ${ }^{4}$, Sabine Maier (i) $^{3}$ \& Konstantin Amsharov (1) ${ }^{1}$

Zig-zag nanographenes are promising candidates for the applications in organic electronics due to the electronic properties induced by their periphery. However, the synthetic access to these compounds remains virtually unexplored. There is a lack in efficient and mild strategies origins in the reduced stability, increased reactivity, and low solubility of these compounds. Herein we report a facile access to pristine zig-zag nanographenes, utilizing an acid-promoted intramolecular reductive cyclization of arylaldehydes, and demonstrate a three-step route to nanographenes constituted of angularly fused tetracenes or pentacenes. The mild conditions are scalable to gram quantities and give insoluble nanostructures in close to quantitative yields. The strategy allows the synthesis of elusive low bandgap nanographenes, with values as low as $1.62 \mathrm{eV}$. Compared to their linear homologues, the structures have an increased stability in the solid-state, even though computational analyses show distinct diradical character. The structures were confirmed by X-ray diffraction or scanning tunneling microscopy.

\footnotetext{
${ }^{1}$ Department of Chemistry and Pharmacy, Organic Chemistry II, Friedrich-Alexander-University Erlangen-Nuernberg, Nikolaus-Fiebiger-Str. 10,91058 Erlangen, Germany. ${ }^{2}$ Department of Chemistry \& Molecular Technology Innovation Presidential Endowed Chair, University of Tokyo, 7-3-1 Hongo, Bunkyoku, Tokyo 113-0033, Japan. ${ }^{3}$ Department of Physics, Friedrich-Alexander-University Erlangen-Nuernberg, Erwin-Rommel-Str. 1, 91058 Erlangen, Germany. ${ }^{4}$ Chemistry Department, Moscow State University, Leninskie Gory, Moscow, Russia 119991. Correspondence and requests for materials should be addressed to K.A. (email: konstantin.amsharov@fau.de)
} 
T he chemistry of polycyclic aromatic hydrocarbons (PAHs), essentially founded by E. Clar in the middle of the 20th century $^{1}$, is nowadays flourishing more than ever in form of structurally precise carbon nanostructures ${ }^{2,3}$. Driven by the vast potential in next generation technologies ${ }^{4-6}$, and accelerated by the continuous development of synthetic methodologies ${ }^{7-12}$, the field of PAHs and nanographenes (NGs) remains intensively studied by researchers from the physical sciences. The high interest origins in the fact that the physical and chemical properties of the hydrocarbons are directly related to their size, shape, and especially edge topology (Fig. 1a) ${ }^{13}$. In that respect, PAHs with zig-zag periphery are especially interesting, because they show typically higher charge carrier mobilities than compounds with an armchair periphery. Further, they exhibit strong absorptions in the visible region as a result of a decreased highest occupied molecular orbital-lowest unoccupied molecular orbital gap (HOMO-LUMO gap; HLG) ${ }^{14,15}$. As a consequence, significant contributions from open-shell resonances in the ground state are frequently observed ${ }^{16}$. Keywords that highlight acenetype PAHs with zig-zag periphery range from singlet fission ${ }^{17-21}$, auspicious charge carrier mobilities ${ }^{22,23}$, to amplified spontaneous emission ${ }^{24}$, and enlarge the interest in PAHs by the fields of spintronics and molecular magnetism ${ }^{25-27}$. Recently, various research groups reported on substituted angularly fusedtetracenes ${ }^{28-32}$, which overcome the low stability of linear acenes, by increasing the intrinsic number of Clar's-sextets ${ }^{33}$. These fused structures showed excellent performances in fieldeffect transistors ${ }^{28,29,31,32}$. Among these slipped bis-tetracenes, a significant focus was set on derivatives of the 2.3,8.9-dibenzanthanthrene (DBATT) aromatic carbon skeleton. Earlier, DBATT was already revealed as a probe for the Shpol'skii effect $^{34-36}$, it was used as single molecule optical transistor ${ }^{37}$, or as solid-state single-quantum emitter ${ }^{38}$. Interestingly, even though this molecule was known since the early explorations of $\mathrm{E}$. Clar in the 1940s, to date its structure has been assigned only by chemical rational and UV/Vis spectroscopy ${ }^{39,40}$. However, repetition of Clar's route in our own laboratories yielded DBATT only in trace amounts and required intensive purification procedures.

Herein, we report on the preparation and investigation of $\pi$ extended molecules based on the DBATT carbon skeleton. We developed a facile three-step synthesis, which utilizes an acidpromoted reductive intramolecular cyclization of aromatic aldehydes - a dehydrative $\pi$-extension (DPEX) reaction - as key transformation. Our DPEX protocol allows the synthesis of DBATT in highly pure form in close to quantitative yield. Importantly, DPEX is suitable for the preparation of pristine zigzag edges, without the need of bulky or electronically stabilizing substituents, which preserves and allows to study the true nature of the zig-zag nanographenes (zzNGs). Finally, the power of DPEX is demonstrated by the preparation of the $\pi$-extended and unprecedented homologues, fused tetrakis-tetracene (TTc), slipped bis-pentacene (BPc), and fused tetrakis-pentacene (TPc); pushing the boundaries of the bottom-up preparation of zzNGs (Fig. 1b).

\section{Results}

Synthesis of nanographenes by DPEX. In the case of DBATT and its derivatives, pyrene was brominated with bromine in chloroform, yielding 1,6-dibromopyrene 1 on a decagram scale, which was purified by a single recrystallization step from xylenes (see Supplementary Methods) ${ }^{41,42}$. Two-fold Suzuki-Miyaura coupling of $\mathbf{1}$ with 2 -formylphenylboronic acid $\mathbf{2}$ gave the diarylated pyrene precursors, 1,6-bis(2-formylphenyl)pyrene 3, which was obtained in pure form and good yields after silica gel

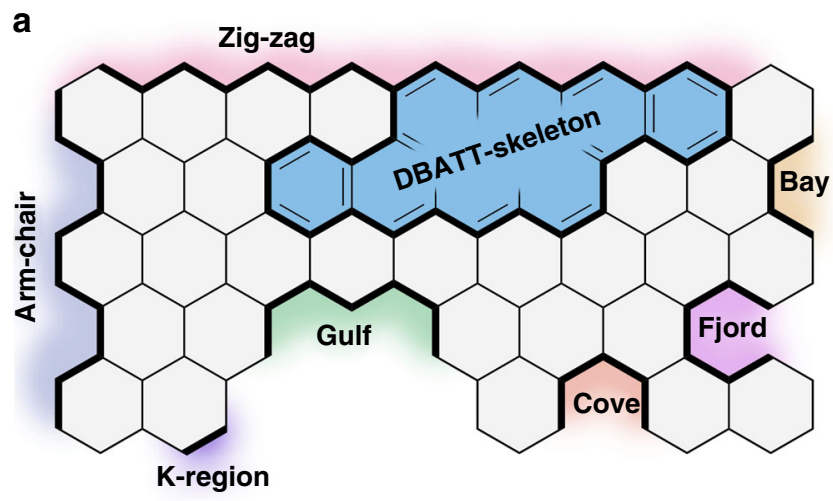

\section{This work}

b

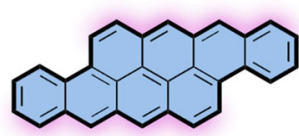

DBATT
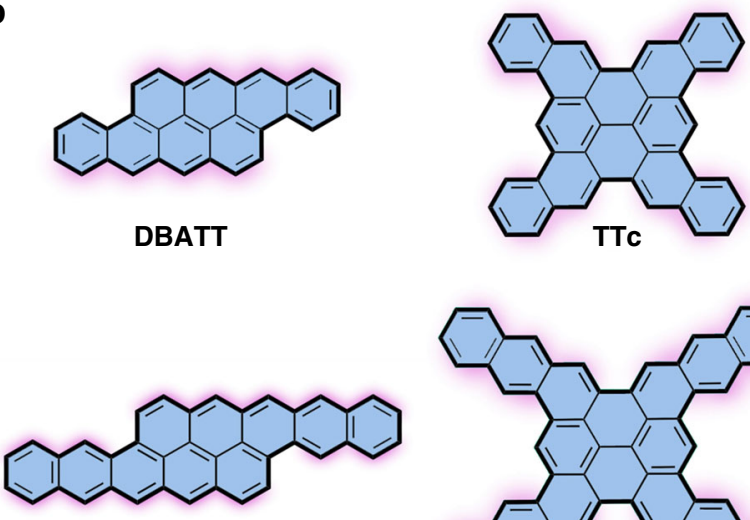

BPc

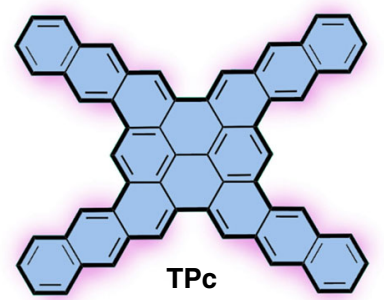

Fig. 1 Relevant terminologies and overview of this work. a Nomenclature and schematic depiction of relevant edge structures; $\mathbf{b}$ Herein enabled zigzag NGs by the DPEX reaction

plug filtration and precipitation with hexanes from dichloromethane. The preparation of BPc-precursor 4, was achieved analogously by the coupling with 3 -formylnaphthalene-2-boronic acid pinacol ester 5, which was obtained in three steps from 1,2,4,5-tetrabromobenzene (see Supplementary Methods). As proof of principle, regioselective 3,8-functionalization of $\mathbf{3}$ could be easily achieved by bromination to $\mathbf{6}$ which can be either directly condensed to dibromo DBATT (bBr-DBATT) via DPEX (see Supplementary Methods) or converted to the respective aryl derivative by Suzuki-Miyaura coupling with e.g., phenylboronic acid to 3a, yielding respective diaryl DBATTs (see also compound $\mathbf{1 6}$ and pyridinyl substituted compound S5). This route avoids the earlier reported formation of non-selective 1,2 and 1,4Michael addition products ${ }^{30,43}$. For small scale brominations, the 1:2 hexamethylentetramine-bromine complex (HMTAB) was found to be particularly useful, however not mandatory for the selective bromination ${ }^{44}$. Starting from 1,3,6,8-tetrabromopyrene $\mathbf{7}$, four-fold-substituted pyrenes $\mathbf{8}$ and $\mathbf{9}$ were obtained in equal manner. An overview on the synthetic scheme is shown in Fig. 2. Precursors 3, 3a, 4, 8, and 9 were subjected to the elaborated DPEX conditions (vide infra, see also Supplementary Table 1), giving the target NGs in close to quantitative yield, without any sign of side-product formation, as determined by HPLC and MS. Supplementary Table 2 shows all synthesized precursors and Supplementary Table 3 shows all nanographenes accessed by 


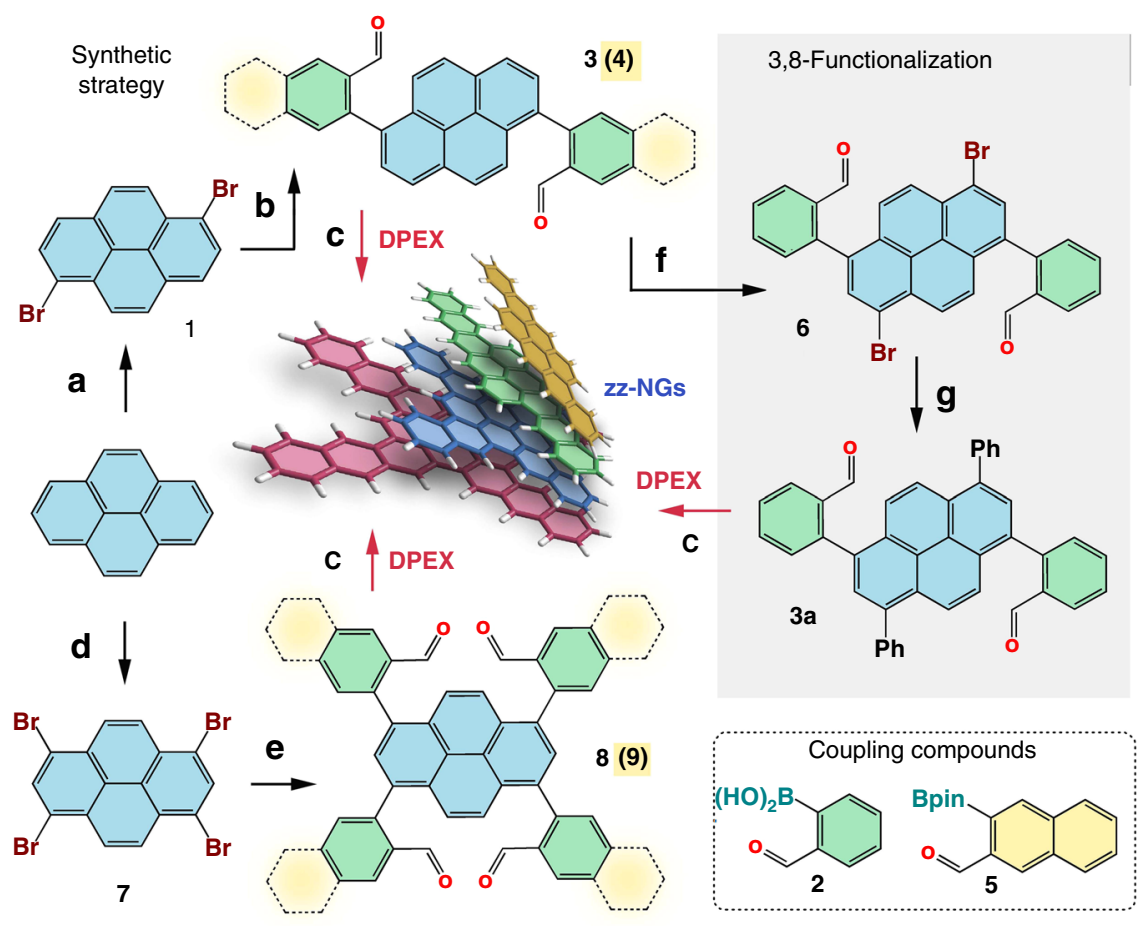

Fig. 2 Synthesis of zig-zag NGs. a $\mathrm{CHCl}_{3}, \mathrm{Br}_{2} ;$ b 2:1 toluene/MeOH, 2.5\% $\mathrm{Pd}\left(\mathrm{PPh}_{3}\right)_{4}, \mathrm{~K}_{2} \mathrm{CO}_{3}, 80{ }^{\circ} \mathrm{C}, \mathrm{N}_{2}(3: 61 \%, 4: 65 \%) ; \mathbf{c} \mathrm{DPEX}: \mathrm{CH} \mathrm{Cl}_{2}, 2$ vol\% sat. $\mathrm{SnCl}_{2} \cdot 2 \mathrm{H}_{2} \mathrm{O} / \mathrm{i}-\mathrm{PrOH}, 1$ vol\% conc. $\mathrm{H}_{2} \mathrm{SO}_{4}$, rt, (quant.); d nitrobenzene, $\mathrm{Br}_{2} ;$ e 2:1 toluene/MeOH, $4 \% \mathrm{Pd}\left(\mathrm{PPh}_{3}\right), \mathrm{K}_{2} \mathrm{CO}_{3}, 80{ }^{\circ} \mathrm{C}, \mathrm{N}_{2}(8: 81 \%, 9: 91 \%) ; \mathbf{f}$ $\mathrm{CH}_{2} \mathrm{Cl}_{2}, 1.6$ equiv. $\mathrm{HMTAB}$, rt (quant.); g 2:1 toluene/MeOH, $2.5 \% \mathrm{Pd}\left(\mathrm{PPh}_{3}\right)_{4}, \mathrm{~K}_{2} \mathrm{CO}_{3}, 80^{\circ} \mathrm{C}_{1} \mathrm{~N}_{2}$ (3a: $66 \%$ with phenylboronic acid); $\mathrm{HMTAB}: 1: 2$ hexamethylentetramine-bromine complex

DPEX (DBATT, bPh-DBATT, bBr-DBATT, TTc, BPc, TPc, 11, 13, 17, and S7). Even though DPEX takes place already in neat $\mathrm{H}_{2} \mathrm{SO}_{4}$ in moderate yields (see Supplementary Table 1), the best possible conditions were first refined by the conversion of 3 to DBATT on an analytical scale. The reaction outcome was followed by quantitative HPLC analysis.

3 and the other nanographene precursors show relatively good solubility in $\mathrm{CH}_{2} \mathrm{Cl}_{2}$ and THF compared to other tested solvents like e.g., hexanes, toluene, and ethyl acetate, which subsequently determined the tested reaction media for the DPEX reaction. However, only $\mathrm{CH}_{2} \mathrm{Cl}_{2}$ as solvent provides the best reaction outcome. As reducing agent, we focused on common $\mathrm{SnCl}_{2} \cdot 2 \mathrm{H}_{2} \mathrm{O}$. The addition of $2 \mathrm{vol} \%$ of a saturated solution of $\mathrm{SnCl}_{2} \cdot 2 \mathrm{H}_{2} \mathrm{O}$, dissolved in iso-propanol is superior over all other investigated reduction systems. Interestingly, using methanol instead, which dissolves $\mathrm{SnCl}_{2} \cdot 2 \mathrm{H}_{2} \mathrm{O}$ by far better than isopropanol, the conversion appears to be faster, however the reaction is accompanied by the formation of unidentified side products. Although the side products are formed in trace amounts, as indicated by HPLC analysis, further post synthetic purification appears to be difficult due to the low solubility. On the other hand, the addition of iso-propanol significantly slows down the conversion but remarkably improves the selectivity and the reaction outcome; used as mere solvent however, the reaction is inhibited completely. The addition of 1 vol\% conc. $\mathrm{H}_{2} \mathrm{SO}_{4}$ initiates the reaction, which is indicated (in case of $\mathbf{3}$ and $\mathbf{3 a}$ ) by a rapid purple coloration of the mixture, accompanied by the formation of a white precipitate. Supplementary Fig. 1 shows a pictured illustration of the single reaction steps. Hereby, the empiric ratio of $2: 1$ of the $\mathrm{SnCl}_{2} / i$ - $\mathrm{PrOH}$-solution and the $\mathrm{H}_{2} \mathrm{SO}_{4}$ plays a crucial role for a successful outcome. After work-up with aqueous hydrochloric acid and extraction, the product is precipitated with $\mathrm{MeOH}$, which gives pure DBATT in close to quantitative yields. The usage of stronger acids like e.g., trifluoromethanesulfonic acid (TfOH), or $34 \%$ oleum shows good performances, however significantly worse than conc. $\mathrm{H}_{2} \mathrm{SO}_{4}$. On the other hand, weaker acids like e.g., trifluoroacetic acid (HTFA) and acetic acid, show no conversion at all. As mentioned above, reactions carried out in neat $\mathrm{H}_{2} \mathrm{SO}_{4}$ and thus lacking a reducing agent were found to give DBATT in moderate yields. Since the required two-electron reduction process cannot stem from $\mathrm{H}_{2} \mathrm{SO}_{4}$, we surmise a disproportionation reaction between two intermediate molecules, leading to DBATT and oxidized derivatives. This assumption is further supported by the fact that the yields of DBATT decreased upon lowering the concentration of 3 and never exceeded yields of 50\%. Further details can be extracted from Supplementary Table 1.

With respect to the scope of the DPEX protocol, the reactivity is demonstrated using the less reactive naphthalene core as the central aromatic unit. The respective precursor molecules $\mathbf{1 0}$ and 12 are obtained from 1,4-dibromonaphthalene and 1,5-diiodonaphthalene by standard two-fold Suzuki-Miyaura coupling reactions (see Supplementary Methods). Despite the lower activity of the naphthalene core, the DPEX cyclization results the desired benzo[rst]pentaphene $\mathbf{1 1}$ and dibenzo[b,def]chrysene 13 in moderate yields (Fig. 3a). Interestingly, in both cases the cyclisation is only successful in the presence of $\mathrm{SnCl}_{2}$, indicating that $\mathrm{SnCl}_{2}$ plays a crucial role in the DPEX process and participates already in the first reaction step. Thus, the mechanism of DPEX appears to be more complex than the intuitively assumed two-step domino reaction. This is also supported by the lack in formation of undesired pentagons under DPEX conditions, which otherwise would be expected for protonated forms of aldehydes. This transformation however, is completely suppressed as demonstrated by the attempt to synthesize indeno[1,2-b]fluorene 15 from $p$-terphenyl dicarbaldehyde 14 (Fig. 3b). Surprisingly, compound 14 remains completely intact under DPEX condition, pointing out that the 
a<smiles>Cc1ccccc1-c1ccc(-c2ccccc2C=O)c2ccccc12</smiles>

10

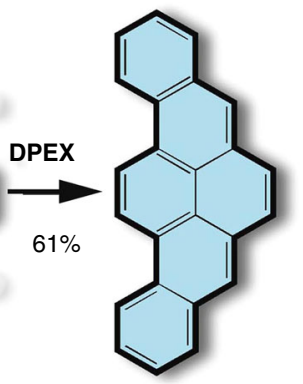

11

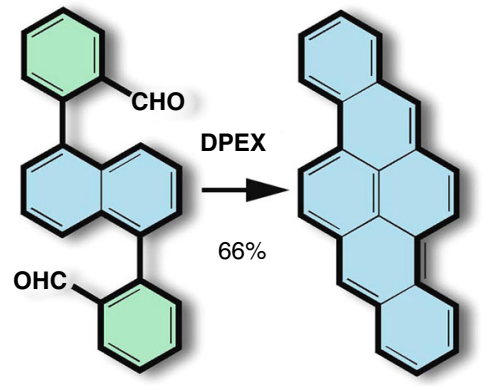

13

b

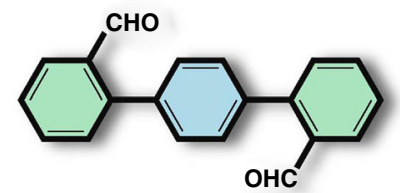

14
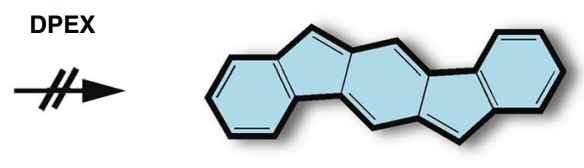

15
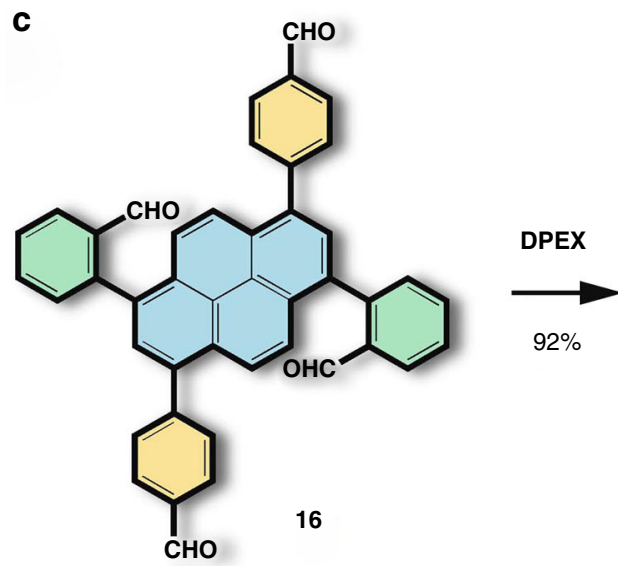

Fig. 3 The scope of the DPEX protocol. a Reaction with less reactive naphthalene core units; $\mathbf{b}$ No formation of five-membered rings; c Tolerance towards misplaced formyl groups

aldehyde functionality tolerates the reaction conditions if being misplaced. In other words, the aldehyde group shows activity only if it is placed in the formal cove region of the PAH. In order to support further this claim, the para-formylphenyl DBATT precursor 16, bearing two aldehyde groups in active, and two in inactive positions was prepared, starting from dibromo precursor 6. As shown in Fig. 3c, 16 reacts under typical DPEX conditions selectively, yielding the desired DBATT derivative 17 in $92 \%$ isolated yield. This unprecedentedly high regioselectivity of DPEX provides essential flexibility in design and facile access to complex functional PAHs. Regarding other functionalities, DPEX shows to be tolerant towards keto-groups, which undergo no transformation (compare compound S6 in the Supplementary Methods); heterocycles like pyridine substituted precursors do not affect the outcome of DPEX and nearly quantitative conversions are obtained (see compound S7 in the Supplementary Methods).

Noteworthy, the solvents were neither degassed, pre-distilled or purified, and used as obtained from the suppliers, which underpins the applicability of this protocol. Moreover, the reactions were performed under ambient atmosphere at room temperature. The only pre-caution taken was the avoidance of direct light irradiation, since DBATT is known to undergo light-induced oxidative decomposition ${ }^{39}$. In order to proof that this reductive-condensation protocol is suitable for the preparative-scale production, we carried out the reaction on a $0.50 \mathrm{~g}$ scale of 3, which allowed us to isolate DBATT in $0.44 \mathrm{~g}$ as pure dark blue solid (isolated yield 96\%). Supplementary Fig. 1 shows a detailed and pictured illustration of the single reaction steps. Accordingly, TTc was prepared on a $100 \mathrm{mg}$ scale and BPc and TPc on a $20 \mathrm{mg}$ scale.

Structure elucidation. Structure analysis of highly insoluble pristine NGs is evolving as major problem in modern nanographene sciences. Therefore, solubilizing groups are typically attached to the PAH's skeleton, leading inevitably to an alteration of its original characteristics ${ }^{28-30,32}$. Spectroscopically at the border of solubility for NMR analysis, we elucidated the ${ }^{1} \mathrm{H}$ NMR spectrum of DBATT, obtained at $100{ }^{\circ} \mathrm{C}$ in $o$-DCB (Fig. 4a). The peaks were assigned by the correlation with its computed NMR spectrum (RB3LYP 6-311 + G(d,p) GIAO). The absorption and emission spectra of DBATT are shown in Fig. 4b. The lowest energy absorption maximum is found at $\lambda_{\max }=586 \mathrm{~nm}$; it is absorption onset of $\lambda_{\text {onset }}=600 \mathrm{~nm}$ corresponds to the HOMO-LUMO transition of $2.07 \mathrm{eV}$ and compares well to its computed HLG (vide infra). The small Stoke's shift of $4 \mathrm{~nm}$ gives an emission maximum at $600 \mathrm{~nm}$ and is characteristic for the rigid carbon skeleton. For the irrefutable structure determination, we took advantage of the stability of our zzNGs towards thermal 
a
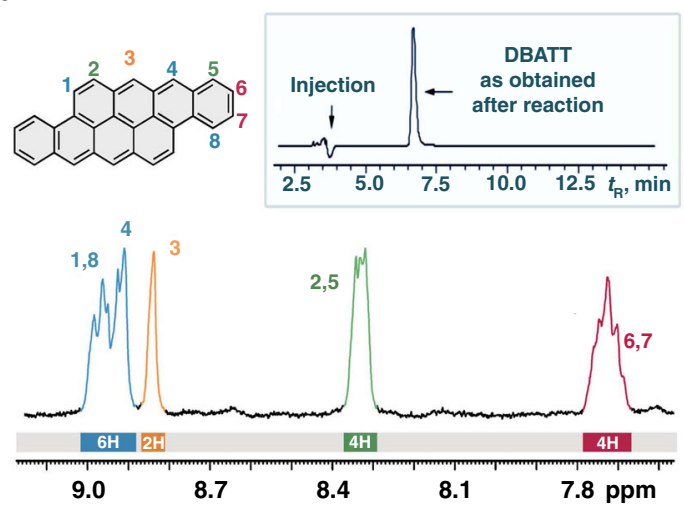

b

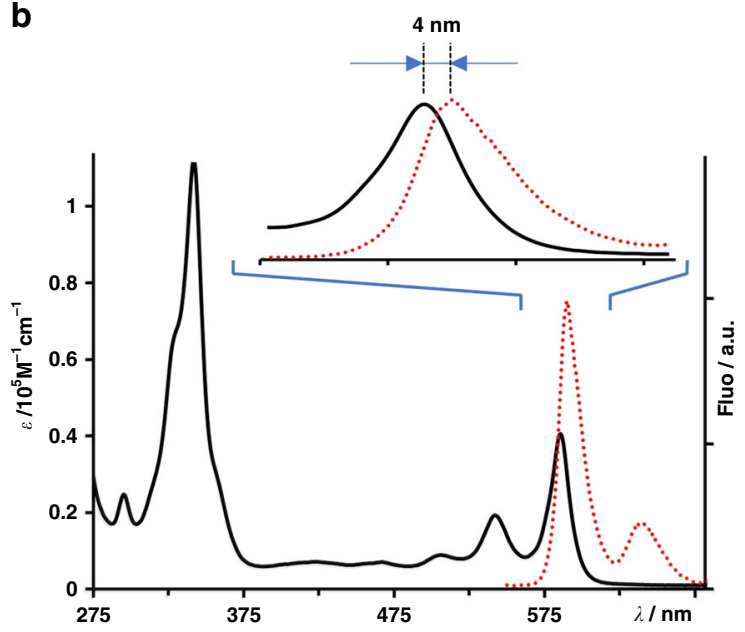

C

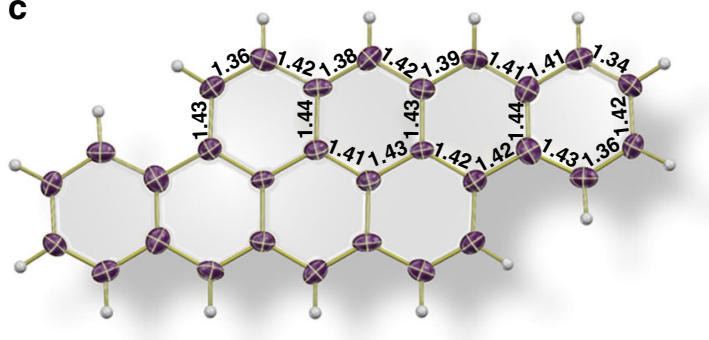

d

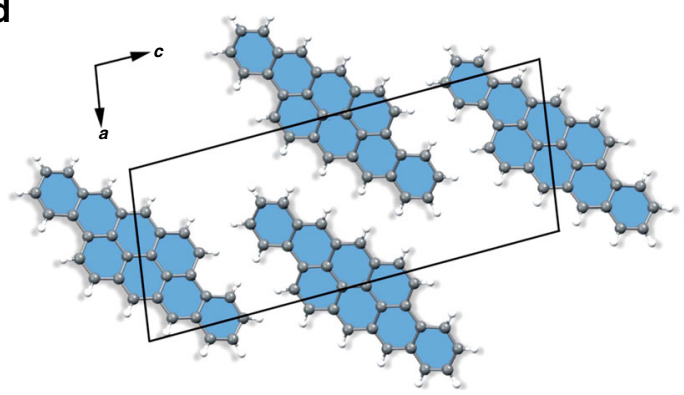

e

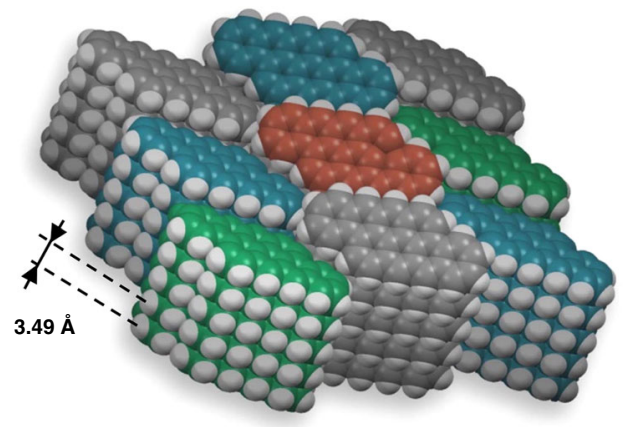

Fig. 4 Spectroscopic analysis and structure elucidation of DBATT. a ${ }^{1} \mathrm{H}$ NMR (o-DCB- $\mathrm{D}_{4}, 100^{\circ} \mathrm{C}, 400 \mathrm{MHz}$; ; proton assignments were correlated with computed NMR spectra at the DFT RB3LYP 6-311 + G(d, p) GIAO level of theory; inset shows HPLC chromatogram after reaction work-up; b absorption (black) and emission (red) in THF at rt; c single crystal X-ray structure depicted as ORTEP model with 50\% thermal ellipsoids, independent C-C bond lengths are indicated; $\mathbf{d}$ View onto the (101) face of the crystal (lattice) structure; depicted as balls and sticks model; e columnar crystal packing motif of DBATT with an interlayer distance of $3.49 \AA$; depicted as space filling model

sublimation. In case of DBATT, we were able to grow crystals in shape of dark blue needles (see Supplementary Fig. 2) by sublimation at $310^{\circ} \mathrm{C}$ at $10^{-5} \mathrm{mbar}$, suitable for single crystal X-ray diffraction (see Fig. 4c). Unlike the examples from substituted DBATT structures ${ }^{28-30,32}$, the aromatic skeleton of pristine DBATT remains flat. However, the lack of substituents becomes most apparent in view of the crystal packing. While e.g., threefold substituted triisopropylsilylethynyl-DBATT ${ }^{30}$, packs in a pseudosandwich herringbone motif with a $\pi-\pi$ distance of $3.61 \AA$, pristine DBATT assembles in slipped co-facially aligned columnar stacks with an interlayer distance of $3.49 \AA$ (Fig. $4 \mathrm{~d}$, e). This particular arrangement is especially favorable for energy efficient exciton splitting processes, known as singlet fission, as the eclipsed conformation maximizes the frontier molecular orbital overlap of the HOMO and the LUMO ${ }^{45,46}$.

With 10,12 , and 16 annulated benzene rings respectively, the actual highlight-NGs - TTc, BPc and TPc - could not be brought into solution without decomposition (boiling 1,2,4-trichlorobenzene). In that respect, laser desorption ionization mass spectrometry, as shown in Fig. $5 \mathrm{a}-\mathrm{c}$, indicate the high selectivity and full completeness of the DPEX process. No starting material, intermediates and other side products can be detected by LDIMS which gave the first evidence for the constitutional integrity of the NGs. In the case of BPc a small intensity signal corresponding to the oxygen adduct can be detected, indicating slow oxidation of the compound under ambient conditions (no special precautions were taken during the MS preparation and analysis).

The structural proof was unambiguously obtained by lowtemperature scanning tunneling microscopy (STM). The NGs were sublimed in ultra-high vacuum between 300 and $395{ }^{\circ} \mathrm{C}$ onto a $\mathrm{Au}(111)$ or $\mathrm{Ag}(111)$ surface, which was kept at room temperature. The STM results shown in Fig. 5d-f reveal that the size and shape of the NGs fit perfectly to the superimposed structural models, which were obtained by DFT. BPc and TPc adsorb as single molecules on $\mathrm{Au}(111)$ and $\mathrm{Ag}(111)$ respectively, while TTc forms a self-assembly on $\mathrm{Au}(111)$ (detailed analysis of the self-assembly is discussed in the Supplementary Discussion and depicted in Supplementary Fig. 14). This is the first report on the successful preparation and characterization of such extended zig-zag nanographenes.

Computations. In order to shed light into the electronic properties of the NGs, we carried out DFT calculations. We determined the HOMO-LUMO levels at the B3LYP-6-311+ G(d,p) level of theory; the theoretical diradical character $y(y=0$ pure closed-shell; $y=1$ pure open-shell) was determined according to 
a

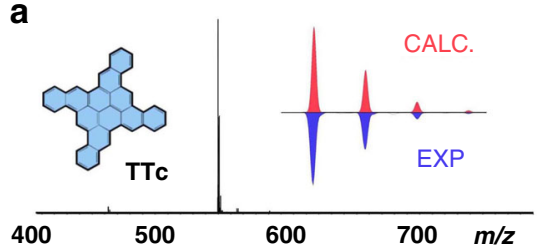

b

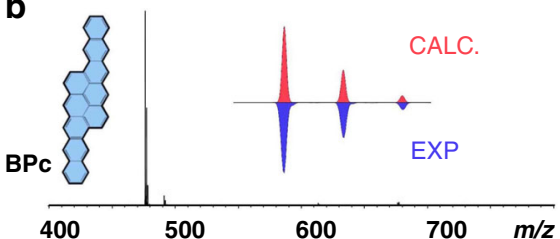

C

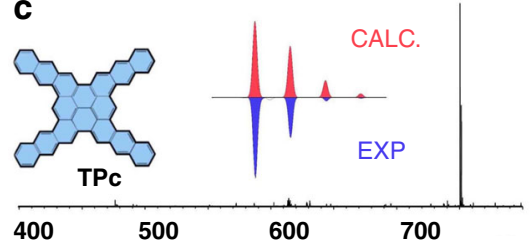

d

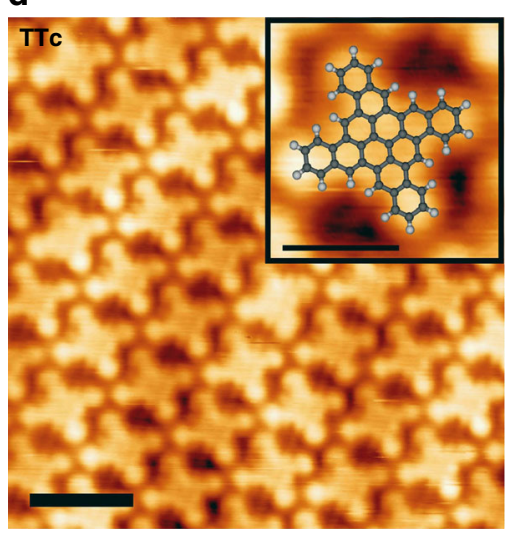

e

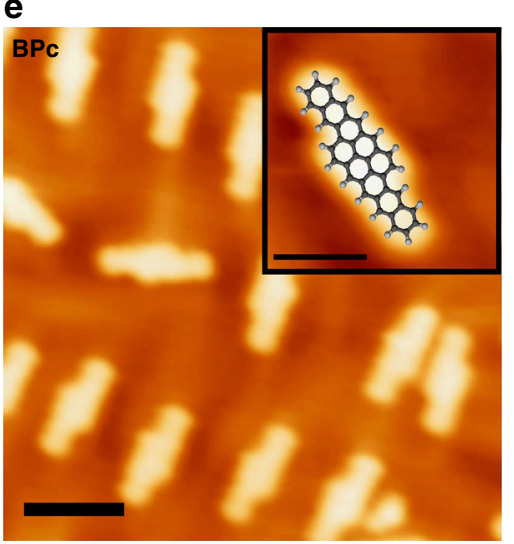

f

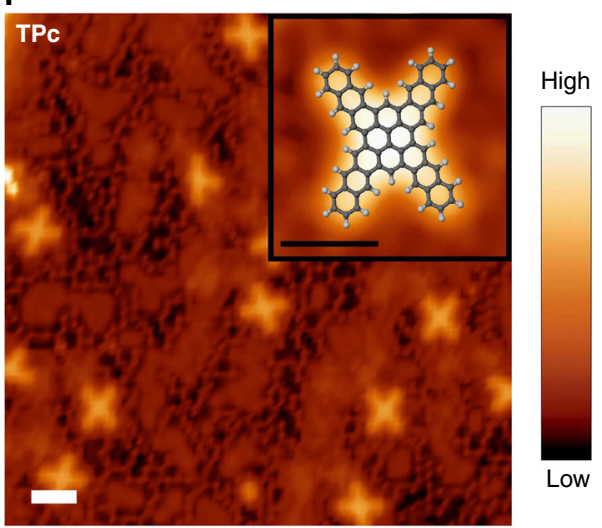

Fig. 5 Structure elucidation of larger NGs - TTc, BPc and TPc. a-c LDI-MS, insets show the calculated and measured isotope pattern, respectively; d-f STM images of TTc on $\mathrm{Au}(111)$ at $77 \mathrm{~K}, \mathbf{B P c}$ on $\mathrm{Au}(111)$ at $4.7 \mathrm{~K}$ and $\mathbf{T P c}$ on $\mathrm{Ag}(111)$ at $4.7 \mathrm{~K}$. The perfect fit of the superimposed DFT models corroborates the unambiguous identification of the NGs. The TPc's are surrounded by bright protrusions that are assigned to halogens, which are residues from the synthesis (see SI). Scale bars: $2 \mathrm{~nm}$ and $1 \mathrm{~nm}$ for the insets with the DFT model overlaid images, respectively. Tunneling conditions: $100 \mathrm{pA} / 1 \mathrm{~V}, 50 \mathrm{pA} /-$ $500 \mathrm{mV}$, and $100 \mathrm{pA} /-500 \mathrm{mV}$

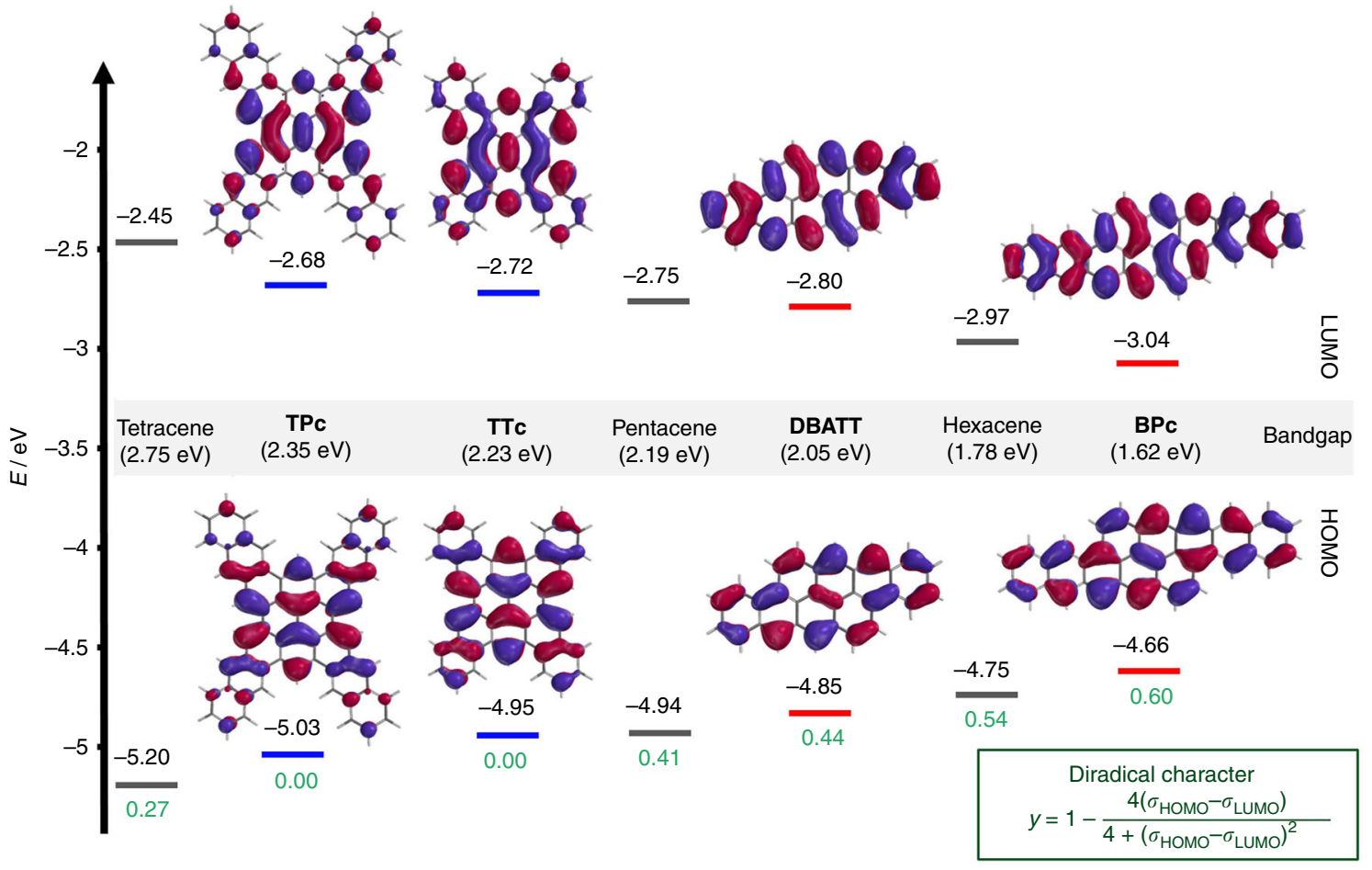

Fig. 6 Computational analysis of the zzNGs. Calculated HOMO-LUMO energies and frontier orbitals (isoval: 0.02 a.u.) at the DFT B3LYP 6-311 G + (d,p) level of theory; Theoretical diradical character $y$ (green digits) was obtained from the broken symmetry calculation at the UHF 6-31+ G(d,p) level of theory

the equation in the inset in Fig. $6^{47}$, from the occupation number of the frontier molecular orbitals $\left(\sigma_{\text {HOMO }}, \sigma_{\text {LUMO }}\right)$, at the UHF $6-31+G(d, p)$ level of theory ${ }^{48,49}$. The values were compared to the parent linear acenes (tetracene, pentacene, and hexacene) and are depicted in Fig. 6. With respect to the HOMO-LUMO gap the cross-shaped NGs TTc and TPc allocate values of $2.23 \mathrm{eV}$ and $2.35 \mathrm{eV}$ between tetracene and pentacene, respectively. At the first glance counterintuitive, TPc reveals a larger gap than TTc. However, this can be attributed to the stabilizing effect of Clar's sextets in the outer benzene rings, which are more dominant in 
TPc and thus lower the HOMO level due to an increased aromatic stabilization energy. Interestingly, from broken symmetry calculations, no mixing of the highest occupied natural orbital (HONO) and lowest unoccupied natural orbital (LUNO), and therefore no diradical character $(y=0)$ for TTc and TPc can be observed. Even though the bandgap of tetracene is bigger than of the respective fourfold fused NGs, it shows already a diradical character of $y=0.27$. Unlike the cross-shaped NGs, the bis-fused compounds DBATT and BPc show, with an increasing number of annulated benzene rings, a decreasing HOMO-LUMO gap (HLG). With a computed gap of $2.05 \mathrm{eV}$, which is in good agreement with its absorption spectrum, DBATT can be located between pentacene and hexacene; with $y=0.44$ it shows a slightly higher diradical character than pentacene. However, in the solidstate DBATT shows to be kinetically much more stable than pentacene, which tends to undergo $[2+2]$ cycloaddition reactions rapidly and requires typically stabilizing groups like e.g., triisopropylsilylacetylene. With a gap of only $1.62 \mathrm{eV}$, which corresponds to a theoretical transition at $765 \mathrm{~nm}$, BPc shows the smallest HLG of the herein discussed NGs and compares well to notoriously unstable heptacene ${ }^{50}$. Even though a significant diradical character of $y=0.60$ is computed for BPc, its persistence during the synthesis, thermal sublimation conditions, and storage in the solid state is remarkable.

\section{Discussion}

The recent progress in nanographene chemistry benefitted to a large extend from synthetic methodologies that allow the design of carbon nanostructures, such as nanographenes (NGs) and graphene nanoribbons, in high structural precision. While the edge-topology displays a strong lever in order to tune the electronic properties of NGs, major achievements towards zzNGs have been achieved only by on-surface synthesis under UHV conditions ${ }^{14}$, or utilizing solubilizing and protective side groups ${ }^{51,52}$. Wet chemically, little synthetic efforts have been made to synthesize pristine zig-zag peripheries. As demonstrated in this study, we developed a facile reaction protocol that allows for the introduction of peripheral zig-zag-methine units into nanographene molecules. The key-step - the dehydrative $\pi$ extension (DPEX) - shows to be, due to its mild conditions, highly efficient in the preparation of nanographenes with small HOMO-LUMO gaps and significant diradical character. Furthermore, we show that the dehydrative cycloaromatization of aldehydes can be realized very effectively; utilizing readily available chemicals, we circumvent the usage of precious transition metals, or otherwise uncommon reagents in the final stage of the synthesis. DPEX showed to be expandable in any direction larger acenes with low bandgaps, and multiple $\mathrm{C}=\mathrm{C}$ bond formations in one molecule, yielding NGs with up to sixteen annulated benzene rings in close to quantitative yield. Its selectivity towards misplaced aldehydes, or ketones, its toleration of halogens, as well as heterocycles like pyridine, allows for a sophisticated compound design. The tolerance of the reaction towards moisture and air, the scalability to gram-quantities, and the extraordinary performance towards the preparation of highly insoluble NGs, makes this methodology a powerful and versatile instrument in the chemists' synthetic toolbox. Thus, we truly believe that alongside the recent synthetic development of synthetic methodologies towards $\mathrm{NGs}^{7-12}$, DPEX will substantially contribute to the blooming field of carbon-based nano-architectures in physical sciences.

\section{Methods}

Synthesis of 2,3,8,9-dibenzanthanthrene DBATT (typical DPEX procedure). A $250 \mathrm{~mL}$ round bottom flask equipped with a magnetic stir bar was charged under ambient atmosphere with precursor $3(20.0 \mathrm{mg}, 48.7 \mu \mathrm{mol})$ and dissolved in
$\mathrm{CH}_{2} \mathrm{Cl}_{2}(100 \mathrm{~mL})$. While stirring, a solution of $\mathrm{SnCl}_{2} \bullet 2 \mathrm{H}_{2} \mathrm{O}(500 \mathrm{mg}, 2.22 \mathrm{mmol})$ in $i$-PrOH $(2.0 \mathrm{~mL})$ was added, followed by the addition of conc. $\mathrm{H}_{2} \mathrm{SO}_{4}(1.0 \mathrm{~mL})$. The mixture was stirred at $\mathrm{rt}$ with protection from daylight for $18 \mathrm{~h}$. The dark purple mixture was quenched by vigorous shaking with $1 \mathrm{M} \mathrm{HCl}(2.0 \mathrm{~mL})$. The mixture was diluted with $\mathrm{CH}_{2} \mathrm{Cl}_{2}(20 \mathrm{~mL})$ and washed with $\mathrm{H}_{2} \mathrm{O}(1 \times 50 \mathrm{~mL})$. The aqueous layer was extracted with $\mathrm{CH}_{2} \mathrm{Cl}_{2}(3 \times 20 \mathrm{~mL})$ including the dark insoluble solids (product). The combined organics were diluted with $\mathrm{MeOH}(100 \mathrm{~mL})$ and the $\mathrm{CH}_{2} \mathrm{Cl}_{2}$ was removed on the rotary evaporator at atmospheric pressure at $50^{\circ} \mathrm{C}$. The formed precipitate in the $\mathrm{MeOH}$ layer was centrifuged, the $\mathrm{MeOH}$ layer was decanted and the solid was washed with $\mathrm{MeOH}$. The product was dried in vacuo and obtained in $98 \%$ yield $(18.0 \mathrm{mg}, 47.8 \mu \mathrm{mol})$ as dark blue solid. ${ }^{1} \mathrm{H}$ NMR (400 MHz, $o$-DCB-D $4,373 \mathrm{~K}): \delta(\mathrm{ppm})=9.02-8.88(6 \mathrm{H}, \mathrm{m}), 8.84(2 \mathrm{H}, \mathrm{m}), 8.38-8.29$ $(4 \mathrm{H}, \mathrm{m}), 7.79-7.67(4 \mathrm{H}, \mathrm{m})$; HRMS (MALDI; DCTB matrix) $(\mathrm{m} / \mathrm{z})$ calculated for $\mathrm{C}_{30} \mathrm{H}_{16}\left(\mathrm{M}^{+}\right)$376.1247, found 376.1764; EA calculated: $\mathrm{C} 95.72, \mathrm{H} 4.28$, found: C 94.88, H 4.54; further details are given in the Supplementary Discussion and Methods.

\section{Data availability}

The data that support the findings of this study are available from the corresponding author upon reasonable request. CCDC 1835109 (DBATT) contains the Supplementary crystallographic data for this paper. These data can be obtained free of charge from The Cambridge Crystallographic Data Centre via www.ccdc.cam.ac. uk/data_request/cif.

Received: 9 July 2018 Accepted: 9 October 2018

Published online: 12 November 2018

\section{References}

1. Clar, E. Polycyclic Hydrocarbons Vol. 1 \& 2 (Springer Verlag, Berlin Heidelberg, 1964).

2. Segawa, Y., Ito, H. \& Itami, K. Structurally uniform and atomically precise carbon nanostructures. Nat. Rev. Mater. 1, 15002 (2016).

3. Narita, A., Wang, X.-Y., Feng, X. \& Müllen, K. New advances in nanographene chemistry. Chem. Soc. Rev. 44, 6616-6643 (2015).

4. Savage, N. Electronics: organic growth. Nature 479, 557-559 (2011).

5. Wu, J., Pisula, W. \& Müllen, K. Graphenes as potential material for electronics. Chem. Rev. 107, 718-747 (2007).

6. Loh, K. P., Tong, S. W. \& Ni, J. Graphene and graphene-like molecules: prospects in solar cells. J. Am. Chem. Soc. 138, 1095-1102 (2016).

7. Koga, Y., Kaneda, T., Saito, Y., Murakami, K. \& Itami, K. Synthesis of partially and fully fused polyaromatics by annulative chlorophenylene dimerization. Science 359, 435-439 (2018)

8. Ito, H., Ozaki, K. \& Itami, K. Annulative $\pi$-Extension (APEX): rapid access to fused arenes, heteroarenes, and nanographenes. Angew. Chem. Int. Ed. 56, 11144-11164 (2017).

9. Seifert, S., Schmidt, D., Shoyama, K. \& Würthner, F. Base-selective five- versus six-membered ring annulation in palladium-catalyzed C-C coupling cascade reactions: new access to electron-poor polycyclic aromatic dicarboximides. Angew. Chem. Int. Ed. 56, 7595-7600 (2017).

10. Seifert, S., Shoyama, K., Schmidt, D. \& Würthner, F. An electron-poor $\mathrm{C}_{64}$ nanographene by palladium-catalyzed cascade $\mathrm{C}$-C bond formation: one-pot synthesis and single-crystal structure analysis. Angew. Chem. Int. Ed. 55, 6390-6395 (2016).

11. Tsvetkov, N. P. et al. Radical alkyne peri-annulation reactions for the synthesis of functionalized phenalenes, benzanthrenes, and olympicene. Angew. Chem. Int. Ed. 57, 3651-3655 (2018).

12. Steiner, A.-K. \& Amsharov, K. Y. The rolling-up of oligophenylenes to nanographenes by HF-zipping approach. Angew. Chem. Int. Ed. 56, 14732-14736 (2017).

13. Ai, Q., Jarolimek, K., Mazza, S., Anthony, J. E. \& Risko, C. Delimited polyacenes: edge topology as a tool to modulate carbon nanoribbon structure, conjugation, and mobility. Chem. Mater. 30, 947-957 (2018).

14. Ruffieux, P. et al. On-surface synthesis of graphene nanoribbons with zigzag edge topology. Nature 531, 489-492 (2016).

15. Anthony, J. E. The larger acenes: versatile organic semiconductors. Angew. Chem. Int. Ed. 47, 452-483 (2008).

16. Zeng, Z. et al. Pro-aromatic and anti-aromatic $\pi$-conjugated molecules: an irresistible wish to be diradicals. Chem. Soc. Rev. 44, 6578-6596 (2015).

17. Lukman, S. et al. Efficient singlet fission and triplet-pair emission in a family of zethrene diradicaloids. J. Am. Chem. Soc. 139, 18376-18385 (2017).

18. Sutton, C., Tummala, N. R., Beljonne, D. \& Brédas, J.-L. Singlet fission in rubrene derivatives: impact of molecular packing. Chem. Mater. 29, 2777-2787 (2017)

19. Basel, B. S. et al. Unified model for singlet fission within a non-conjugated covalent pentacene dimer. Nat. Commun. 8, 15171 (2017). 
20. Hetzer, C., Guldi, D. \& Tykwinski, R. R. Pentacene dimers as a critical tool for the investigation of intramolecular singlet fission. Chem. Eur. J. 24, 8245-8257 (2018).

21. Pace, N. A. et al. Dynamics of singlet fission and electron injection in selfassembled acene monolayers on titanium dioxide. Chem. Sci. 9, 3004-3013 (2018).

22. Li, J. et al. Aromatic extension at 2,6-positions of anthracene toward an elegant strategy for organic semiconductors with efficient charge transport and strong solid state emission. J. Am. Chem. Soc. 139, 17261-17264 (2017).

23. Liu, J. et al. High mobility emissive organic semiconductor. Nat. Commun. 6, 10032 (2016).

24. Paternò, G. M. et al. Synthesis of dibenzo[hi,st]ovalene and its amplified spontaneous emission in a polystyrene matrix. Angew. Chem. Int. Ed. 56, 6753-6757 (2017).

25. Li, Y., Jia, Z., Xiao, S., Liu, H. \& Li, Y. A method for controlling the synthesis of stable twisted two-dimensional conjugated molecules. Nat. Commun. 7, 11637 (2016).

26. Desroches, M. et al. Breaking bonds and forming nanographene diradicals with pressure. Angew. Chem. Int. Ed. 56, 16212-16217 (2017).

27. Rudebusch, G. E. et al. Diindeno-fusion of an anthracene as a design strategy for stable organic biradicals. Nat. Chem. 8, 753-759 (2016).

28. Sbargoud, K. et al. Low bandgap bistetracene-based organic semiconductors exhibiting air stability, high aromaticity and mobility. Chem. Eur. J. 23, 5076-5080 (2017).

29. Wang, Z. et al. A novel angularly fused bistetracene: facile synthesis, crystal packing and single-crystal field effect transistors. J. Mater. Chem. C 5, 1308-1312 (2017).

30. Reus, C. et al. Unexpected michael additions on the way to 2.3,8.9dibenzanthanthrenes with interesting structural properties. Chem. Eur. J. 22, 9097-9101 (2016).

31. Zhang, L. et al. Bistetracene: an air-stable, high-mobility organic semiconductor with extended conjugation. J. Am. Chem. Soc. 136, 9248-9251 (2014)

32. Broman, S. L. et al. Tetraceno[2,1,12,11-opqra]tetracene-extended tetrathiafulvalene - redox-controlled generation of a large $\mathrm{PAH}$ core. Org. Biomol. Chem. 15, 807-811 (2017).

33. Zhang, L. et al. Unconventional, Chemically stable, and soluble twodimensional angular polycyclic aromatic hydrocarbons: from molecular design to device applications. Acc. Chem. Res. 48, 500-509 (2015).

34. Siyushev, P., Stein, G., Wrachtrup, J. \& Gerhardt, I. Molecular photons interfaced with alkali atoms. Nature 509, 66-70 (2014).

35. Gerhardt, I., Wrigge, G., Hwang, J., Zumofen, G. \& Sandoghdar, V. Coherent nonlinear single-molecule microscopy. Phys. Rev. A 82, 063823 (2010).

36. Gu, Y., Huang, L., Martin, O. J. F. \& Gong, Q. Resonance fluorescence of single molecules assisted by a plasmonic structure. Phys. Rev. B 81, 193103 (2010).

37. Hwang, J. et al. A single-molecule optical transistor. Nature 460, 76-80 (2009).

38. Yang, B., Trebbia, J. B., Baby, R., Tamarat, P. \& Lounis, B. Optical nanoscopy with excited state saturation at liquid helium temperatures. Nat. Photon. 9, 658-662 (2015).

39. Clar, E. Untersuchungen über die Feinstruktur des Anthanthrens und seiner Benzologen nach dem Anellierungsverfahren (Aromatische Kohlenwasserstoffe, XXXIX. Mitteil). Chem. Ber. 4, 328-333 (1943).

40. Clar, E. \& Willicks, W. Aromatische Kohlenwasserstoffe, LXXI. Mitteil.: 2.3;4.5-Dibenzanthanthren, $4^{\prime} .5$-o-phenylen-[naphtho2'.3':3.4-pyren] und 2.3;4.5-dibenz-[1.12-o-phenylen]-anthanthren. Chem. Ber. 3, 743-748 (1956).

41. Grimshaw, J. \& Trocha-Grimshaw, J. Characterisation of 1,6- and 1,8dibromopyrenes. J. Chem. Soc. Perkin Trans. 1, 1622-1623 (1972).

42. Figueira-Duarte, T. M. \& Müllen, K. Pyrene-based materials for organic electronics. Chem. Rev. 111, 7260-7314 (2011).

43. Rao, M. R., Johnson, S. \& Perepichka, D. F. Aromatization of benzannulated perylene-3,9-diones: unexpected photophysical roperties and reactivity. Org. Lett. 18, 3574-3577 (2016).

44. Heravi, M. M., Abdolhosseini, N. \& Oskooie, H. A. Regioselective and highyielding bromination of aromatic compounds using hexamethylenetetramine-bromine. Tetrahedron Lett. 46, 8959-8963 (2005).
45. Buchanan, E. A. \& Michl, J. Packing guidelines for optimizing singlet fission matrix elements in noncovalent dimers. J. Am. Chem. Soc. 139, 15572-15575 (2017).

46. Smith, M. B. \& Michl, J. Recent advances in singlet fission. Annu. Rev. Phys. Chem. 64, 361-386 (2013).

47. Dral, P. O. \& Clark, T. Semiempirical UNO-CAS and UNO-CI: method and applications in nanoelectronics. J. Phys. Chem. A 115, 11303-11312 (2011).

48. Kamada, K. et al. Singlet diradical character from experiment. J. Phys. Chem. Lett. 1, 937-940 (2010).

49. Minami, T. \& Nakano, M. Diradical character view of singlet fission. J. Phys. Chem. Lett. 3, 145-150 (2012).

50. Einholz, R. et al. Heptacene: characterization in solution, in the solid state, and in films. J. Am. Chem. Soc. 139, 4435-4442 (2017).

51. Ni, Y. et al. A peri-tetracene diradicaloid: synthesis and properties. Angew. Chem. Int. Ed. 57, 9697-9701 (2018).

52. Gu, Y., Wu, X., Gopalakrishna, T. Y., Phan, H. \& Wu, J. Graphene-like molecules with four zigzag edges. Angew. Chem. Int. Ed. 57, 6541-6545 (2018).

\section{Acknowledgements}

We thank the Deutsche Forschungsgemeinschaft (DFG-SFB 953 Synthetic Carbon Allotropes, Projects A6, B4, AM-407). D.L. thanks the graduate school of Molecular Science (GSMS) for financial support and the Alexander von Humboldt foundation and the Japan Society for the Promotion of Science (JSPS) for a fellowship. S.M. acknowledges funding from the European Research Council (ERC-2014-StG SURFLINK No. 637831). Helen Hölzel is thanked for the donation of the HMTAB complex.

\section{Author contributions}

K.A. and D.L. conceived the concept of the project. D.L., M.F., and O.P. carried out the experiments. S.T. performed X-ray crystal structure analysis. J.L., M.D., and S.M. conducted the structure determination by low-temperature STM. D.L. conducted the computations. D.L. and K.A. prepared the manuscript with feedback from others.

\section{Additional information}

Supplementary Information accompanies this paper at https://doi.org/10.1038/s41467018-07095-z.

Competing interests: The authors declare no competing interests.

Reprints and permission information is available online at http://npg.nature.com/ reprintsandpermissions/

Publisher's note: Springer Nature remains neutral with regard to jurisdictional claims in published maps and institutional affiliations.

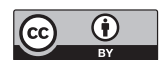

Open Access This article is licensed under a Creative Commons Attribution 4.0 International License, which permits use, sharing, adaptation, distribution and reproduction in any medium or format, as long as you give appropriate credit to the original author(s) and the source, provide a link to the Creative Commons license, and indicate if changes were made. The images or other third party material in this article are included in the article's Creative Commons license, unless indicated otherwise in a credit line to the material. If material is not included in the article's Creative Commons license and your intended use is not permitted by statutory regulation or exceeds the permitted use, you will need to obtain permission directly from the copyright holder. To view a copy of this license, visit http://creativecommons.org/ licenses/by/4.0/.

(C) The Author(s) 2018 